\title{
Overview of Gene Targeting by Homologous Recombination
}

The analysis of mutant organisms and cell lines has been important in determining the function of specific proteins. Until recently, mutants were produced by mutagenesis followed by selection for a particular phenotypic change. Recent technological advances in gene targeting by homologous recombination in mammalian systems enable the production of mutants in any desired gene (Mansour, 1990; Robertson, 1991; Zimmer, 1992). This technology can be used to produce mutant mouse strains and mutant cell lines. Because most mammalian cells are diploid, they contain two copies, or alleles, of each gene encoded on an autosomal (nonsex) chromosome. In most cases, both alleles must be inactivated to produce a discernible phenotypic change in a mutant. The conversion from heterozygosity to homozygosity is accomplished by breeding in the case of mouse strains and by direct selection in cell lines.

Bacteriophage recombinases such as Cre and its recognition sequence, $\operatorname{lox} P$, have also allowed spatial control of knockouts. Another recombinase system, the yeast Flp/FRT system, can also be used (Fiering et al., 1993, 1999). The control can function along actual spatial coordinates when a viral gene transfer system is used, or in a cell type- or tissue-specific fashion when restricted promoters are employed. Adding temporal regulation of Cre, such as that achievable with the tetracyclineregulatable expression system (see CPMB UNIT 16.21 and APPENDIX $1 A$ in this manual), allows temporal control as well.

To produce a mutant mouse strain by homologous recombination, two major elements are needed. An embryonic stem (ES) cell line capable of contributing to the germ line, and a targeting construct containing target-gene sequences with the desired mutation. Maintaining ES cells in their undifferentiated state is a major task during gene targeting (see CPMB UNIT 23.3 and APPENDIX $1 A$ in this manual). This usually is accomplished by growing cells on a layer of feeder cells (see CPMB UNIT 23.2 and APPENDIX 1A in this manual). The targeting construct is then transfected into cultured ES cells (see UNIT 4.30). ES cell lines are derived from the inner cell mass of a blastocyst-stage embryo. Homologous recombination occurs in a small number of the transfected cells, resulting in introduc- tion of the mutation present in the targeting construct into the target gene. Once identified, mutant ES cell clones can be microinjected into a normal blastocyst in order to produce a chimeric mouse. Because many ES cell lines retain the ability to differentiate into every cell type present in the mouse, the chimera can have tissues, including the germ line, with contribution from both the normal blastocyst and the mutant ES cells. Breeding germ-line chimeras yields animals that are heterozygous for the mutation introduced into the ES cell, and that can be interbred to produce homozygous mutant mice.

Homologous recombination can also be used to produce homozygous mutant cell lines. Previously, inactivation of both alleles of a gene required two rounds of homologous recombination and selection (te Riele et al., 1990; Cruz et al., 1991; Mortensen et al., 1991). Now, however, inactivation of both alleles of many genes requires only a single round of homologous recombination using a single targeting construct (Mortensen et al., 1992). The homozygous mutant cells can then be analyzed for phenotypic changes to determine the function of the gene.

\section{ANATOMY OF TARGETING CONSTRUCTS}

Two basic configurations of constructs are used for homologous recombination: insertion constructs and replacement constructs (Fig. 4.29.1). Each can be used for different purposes in specific situations, as discussed below. The insertion construct contains a region of homology to the target gene cloned as a single continuous sequence, and is linearized by cleavage of a unique restriction site within the region of homology. Homologous recombination introduces the insertion construct sequences into the homologous site of the target gene, interrupting normal target-gene structure by adding sequences. As a result, the normal gene can be regenerated from the mutated target gene by an intrachromosomal recombination event.

The replacement construct is the second, more commonly used construct. It contains two regions of homology to the target gene located on either side of a mutation (usually a positive selectable marker; see below). Homologous
Gene Cloning, Expression, and Mutagenesis

\subsection{1}

Supplement 21 


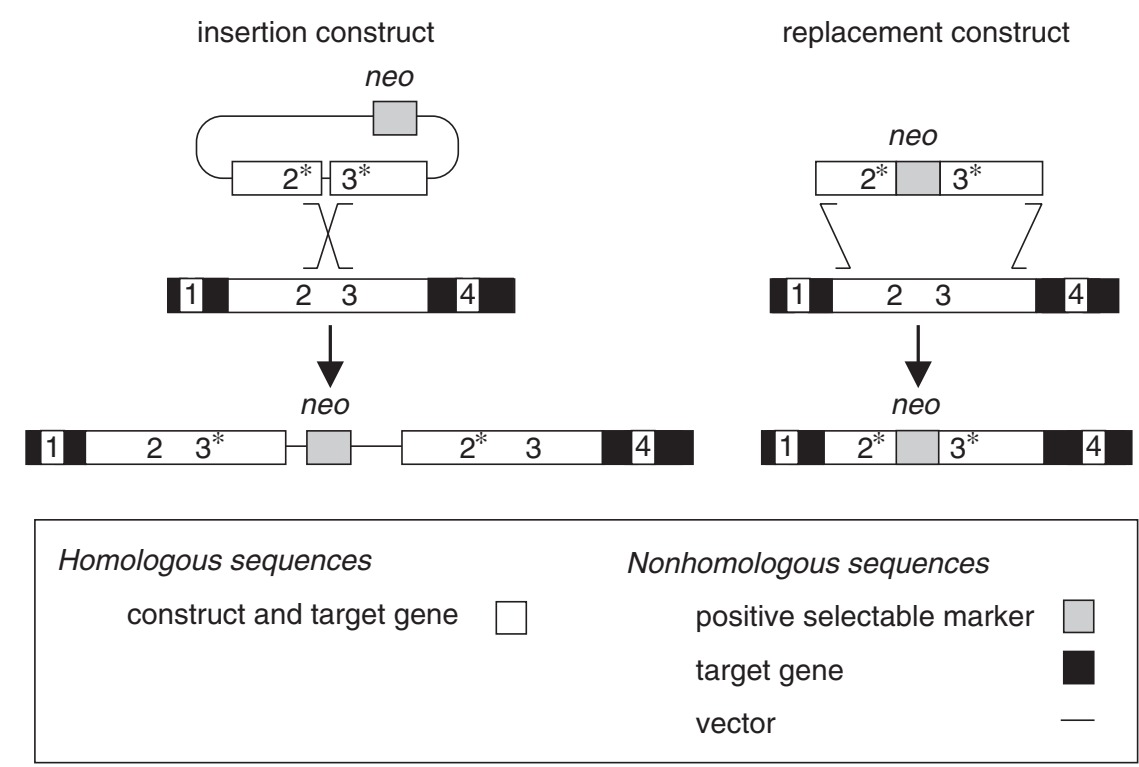

Figure 4.29.1 Two configurations of constructs used for homologous recombination. Numbers indicate target-gene sequences in the genome. An asterisk indicates homologous target-gene sequences in the construct. Replacement constructs substitute their sequences $\left(2^{*}\right.$, neo, and $\left.3^{*}\right)$ for the endogenous target-gene sequences ( 2 and 3$)$. Insertion constructs add their sequences $\left(2^{*}\right.$, $n e o$, and $3^{*}$ ) to the endogenous target gene, resulting in tandem duplication and disruption of the normal gene structure.

recombination proceeds by a double cross-over event that replaces the target-gene sequences with the replacement-construct sequences. Because no duplication of sequences occurs, the normal gene cannot be regenerated.

\section{METHODS OF ENRICHMENT FOR HOMOLOGOUS RECOMBINANTS}

\section{Positive Selection by Drug-Resistance Gene}

Nearly all constructs used for homologous recombination rely on the positive selection of a drug-resistance gene (e.g., neomycin or neo) that is also used to interrupt and mutate the target gene. When either insertion or replacement constructs are linearized, the drugresistance gene is flanked by two regions of homology to the target gene. Selection of the cells using drugs (e.g., G418) eliminates the great majority of transformants that have not stably incorporated the construct (see CPMB UNIT 9.5 and APPENDIX $1 A$ in this manual). However, in many of the surviving clones the construct has incorporated into the genome not by homologous recombination but rather through random integration. Therefore, methods to enrich for homologous recombinant clones have been developed.

\section{Positive-Negative Selection}

The most commonly used method for eliminating cells in which the construct integrated into the genome randomly, thus further enriching for homologous recombinants, is known as positive-negative selection. It is only applicable to replacement constructs (Fig. 4.29.2; Mansour et al., 1988). In these constructs, a negative selectable marker (e.g., herpes simplex virus thymidine kinase, HSV-TK) is included outside the region of homology to the target gene. In the presence of the TK gene, the cells are sensitive to acyclovir and its analogs (e.g., gancyclovir, GANC). The HSV-TK enzyme activates these drugs, resulting in their incorporation into growing DNA, causing chain termination and cell death. During homologous recombination, sequences outside the regions of homology to the target gene are lost due to crossing over. In contrast, during random integration all sequences in the construct tend to be retained because recombination usually occurs at the ends of the construct. The presence of the TK gene can be selected against by growing the cells in gancyclovir; the homologous recombinants will be G418-resistant and gancyclovirresistant, whereas clones in which the construct integrated randomly will be G418-resistant and gancyclovir-sensitive. In some cases, TK is

\subsection{2}




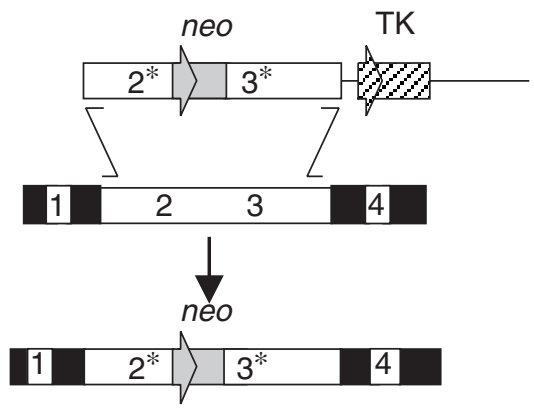

cell phenotype: $\mathrm{G}_{418^{\mathrm{R}}}$

GANC $^{R}$
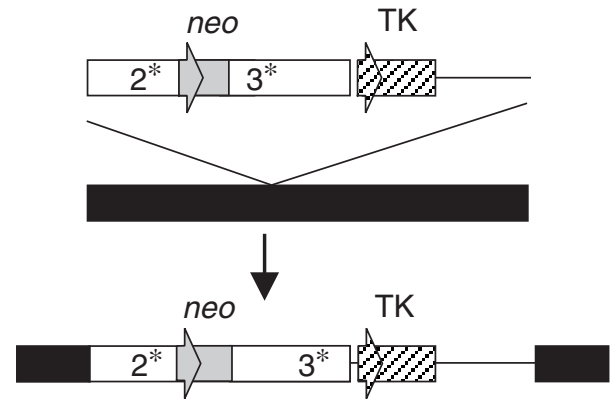

$\mathrm{G} 418^{\mathrm{R}}$

GANCS $^{\mathrm{S}}$

Homologous sequences

construct and target gene
Nonhomologous sequences

positive selectable marker

target gene

negative selectable marker

vector

promoter

Figure 4.29.2 Enrichment for homologous recombinants by positive-negative selection using the TK gene. Homologous recombination involving cross-overs on either side of the neo gene results in loss of the TK gene. Random integration tends to preserve the TK gene. The presence of TK can be selected against because any cell expressing the gene will be killed by gancyclovir (GANC). Although both homologous recombinants and clones in which the construct integrated randomly are G418-resistant, only homologous recombinants are gancyclovir-resistant. The construct is shown linearized so that the plasmid vector sequences remain attached to the TK gene. This configuration helps preserve the integrity of the TK gene. The superscript $R$ denotes resistance and the superscript $\mathrm{S}$ denotes sensitivity.

inactivated without homologous recombination; thus, the gancyclovir-resistant clones must be screened to identify the true homologous recombinants. Other markers that are lethal to cells have also been used instead of TK and gancyclovir (e.g., diphtheria toxin; Yagi et al., 1990).

\section{Endogenous Promoters}

Constructs that rely on an endogenous promoter to express the positive selectable marker can also give enrichment of homologous recombinants (Fig. 4.29.3), but can only be used if the gene of interest is expressed in the cell line. They contain the coding region of a selectable marker (e.g., neo) but lack a promoter for the marker. The coding sequence for the marker usually interrupts, and is in frame with, an exon of the target gene. Thus, when homologous recombination occurs, a fusion protein is produced, driven by the endogenous target-gene promoter. In contrast, when random integration occurs, the selectable-marker protein is not usually produced. Therefore, homologous recombinants are G418-resistant, whereas cells in which the construct integrated randomly are G418-sensitive. Constructs containing a promoterless selectable marker can be constructed in either replacement or insertion structure and can result in dramatic enrichment for homologous recombinants.

\section{TYPES OF MUTATIONS}

\section{Gene Inactivation}

Homologous recombination has most often been used to completely inactivate a gene (commonly termed "knockout"). Usually, an exon
Gene Cloning, Expression, and Mutagenesis

\subsection{3}

Supplement 21 
homologous recombination

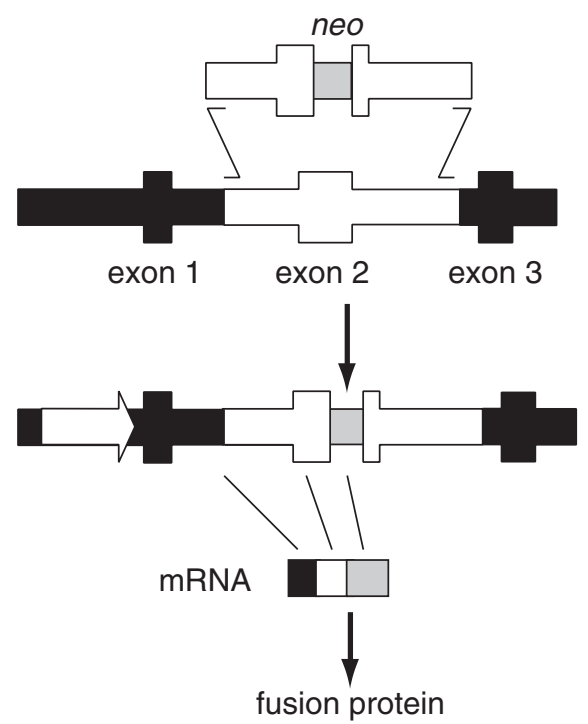

random integration
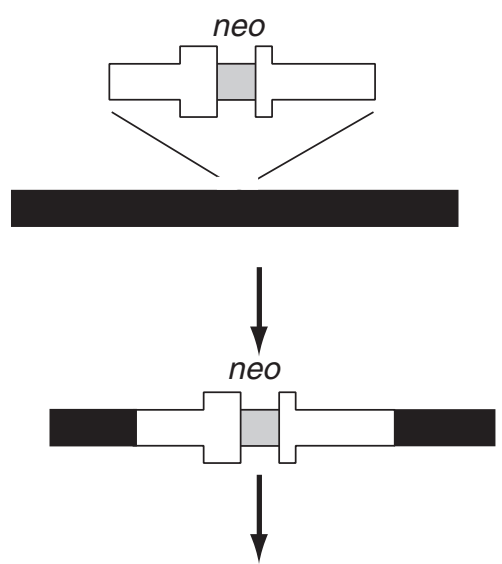

no product

cell phenotype: $\quad \mathrm{G} 418^{\mathrm{R}}$

G418

\begin{tabular}{cc|} 
Homologous sequences & Nonhomologous sequences \\
construct and target gene $\square$ & positive selectable marker $\square$ \\
target gene & promoter
\end{tabular}

Figure 4.29.3 Enrichment for homologous recombinants using a positive selectable marker (neo) lacking a promoter. Clones in which integration of the construct provides an endogenous promoter to drive neo expression will be G418-resistant. The construct is designed so that homologous recombination will provide a promoter leading to neo expression, whereas random integration will most likely not provide a promoter, thus precluding neo expression.

encoding an important region of the protein (or an exon $5^{\prime}$ to that region) is interrupted by a positive selectable marker (e.g., neo), preventing the production of normal mRNA from the target gene and resulting in inactivation of the gene.

A gene may also be inactivated by creating a deletion in part of a gene, or by deleting the entire gene. By using a construct with two regions of homology to the target gene that are far apart in the genome, the sequences intervening the two regions can be deleted. Up to $15 \mathrm{~kb}$ have been deleted in this way; thus, many genes could be completely eliminated (Mombaerts et al., 1991). Gene inactivations may also be controlled using the Cre/lox $P$ recombinase system either spatially, as in cell type- or tissue-specific knockout, or temporally, through control of the activity or expression of the recombinase (see Cre/loxP System, below).
Mutations can be introduced that have multiple purposes. Homologous recombination has been used to introduce a replacement construct containing the coding sequence of $\beta$-galactosidase in frame with the $5^{\prime}$ end of the target gene. Downstream of the $l a c Z$ gene is a positive selectable marker driven by a heterologous promoter (Fig. 4.29.4). This construct not only disrupts target-gene function but also expresses a fusion protein with $\beta$-galactosidase activity, and thus can be used to monitor the activity of the endogenous gene's promoter in various tissues during development (Mansour et al., 1990).

\section{Subtle Gene Mutations}

Homologous recombination can also be used to introduce subtle mutations in a gene. One method is analogous to a method in yeast
Targeting by

Homologous Recombination 

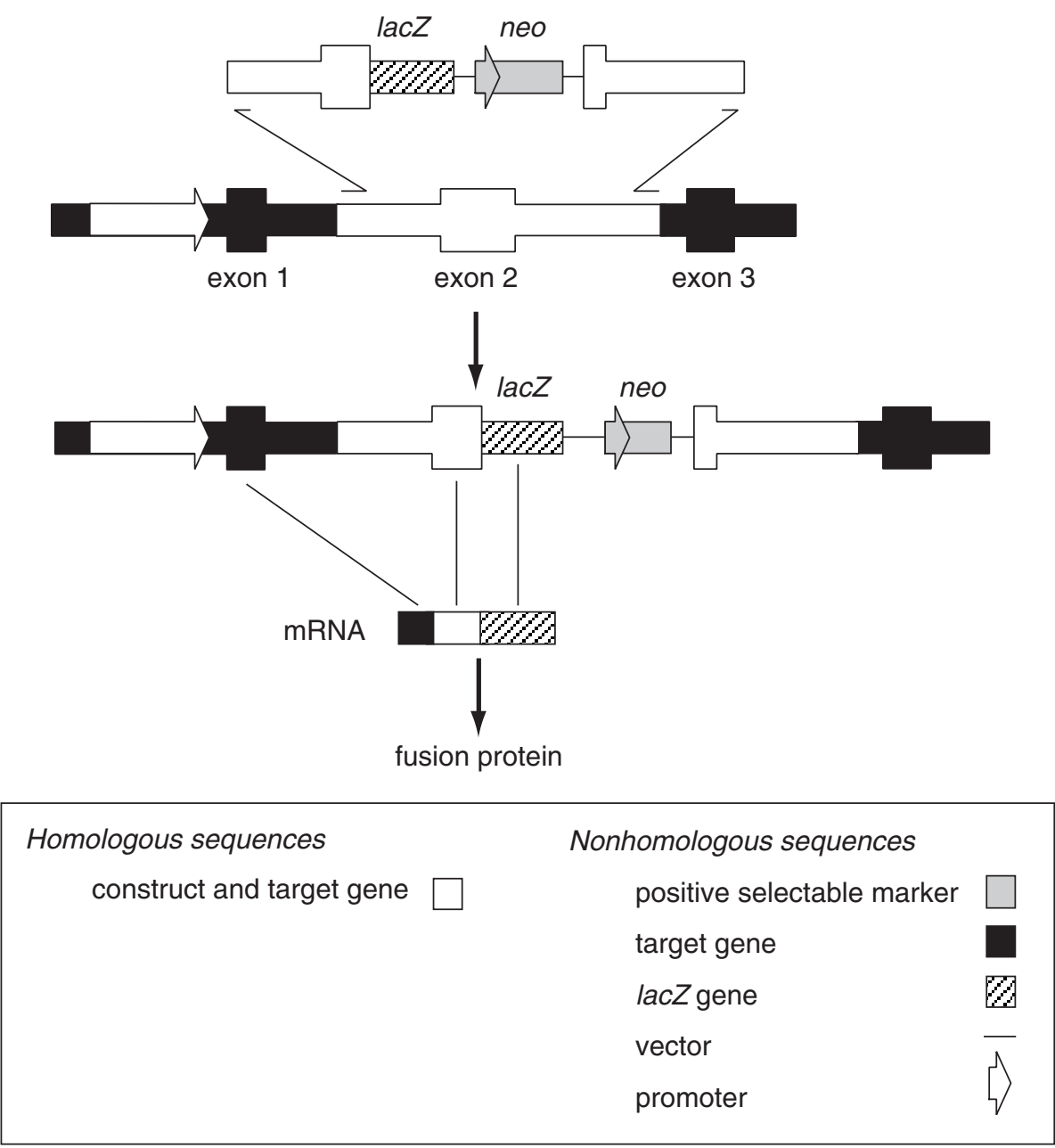

Figure 4.29.4 LacZ reporter construct for gene targeting. This construct has two purposes: first, to disrupt the target gene and, second, to express the lacZ gene as a marker to monitor activity of the endogenous target gene's promoter.

called transplacement or allele replacement ( CPMB UNIT 13.10 and APPENDIX $1 A$ in this manual) because duplications are introduced into the target gene and then removed. An insertion construct containing both positive and negative selectable markers (e.g., neo and TK) is used to introduce a duplication that contains a subtle mutation, such as a point mutation, into the target gene sequence (Fig. 4.29.5). After selection for integration of the construct using the positive selectable marker (e.g., G418), homologous recombinants are identified by screening. A homologous recombinant clone is cultured and then the presence of the negative selectable marker is selected against (e.g., selection against TK using gancyclovir). This selects for an intrachromosomal recombination that eliminates the target-gene duplications and the selectable markers but leaves the mutant target-gene sequences substituting for the normal target-gene sequences. Surviving clones are screened for the correct intrachromosomal rearrangements, leaving the desired mutation. A second method of introducing subtle mutations into a gene is to insert the mutation by homologous recombination and then use the Cre/loxP system to remove the selectable marker.

\section{CRE/loxP SYSTEM}

The Cre/loxP system is derived from the bacteriophage $\mathrm{P} 1$. The recombinase $\mathrm{Cre}$ acts on the DNA site loxP. If there are two loxP sites in the same orientation near each other, Cre can act to loop out the sequence between the two sites, leaving a single lox $P$ site in the original
Gene Cloning, Expression, and Mutagenesis

\subsection{5}

Supplement 22 

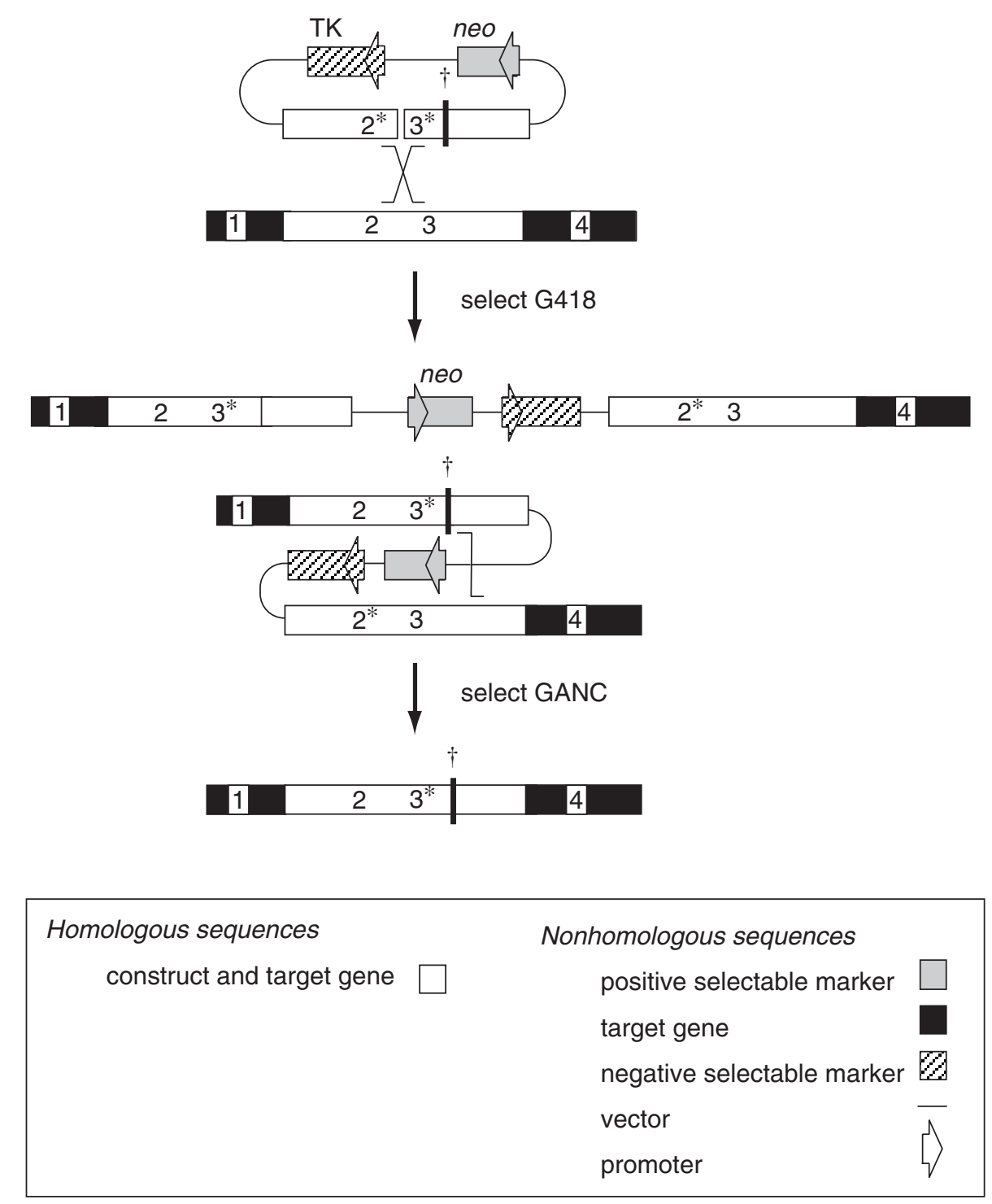

Figure 4.29.5 Allele replacement "hit and run." Cells are cultured in G418 to select for the integration of neo, then homologous recombinants are identified by screening and are cultured in gancyclovir (GANC) to select against the presence of the TK gene. This strategy may yield a reconstituted gene containing the subtle mutation present in the construct (indicated by the dark bar and $\dagger$ ). Because intrachromosomal recombination may result in the loss of the subtle mutation, its presence must be verified (e.g., by a change in restriction site).

Overview of Gene Targeting by

Homologous Recombination

4.29.6

\section{Removing the Positive Selectable Marker}

Although many gene inactivation approaches involving homologous recombination still use constructs that leave the positive selectable marker in the genomic DNA, it has become increasingly clear that this can cause a number of unanticipated effects. For example, the presence of the neo gene, often with its own promoter, can alter the expression of neighboring loci (Olson et al., 1996; Pham et al., 1996). 


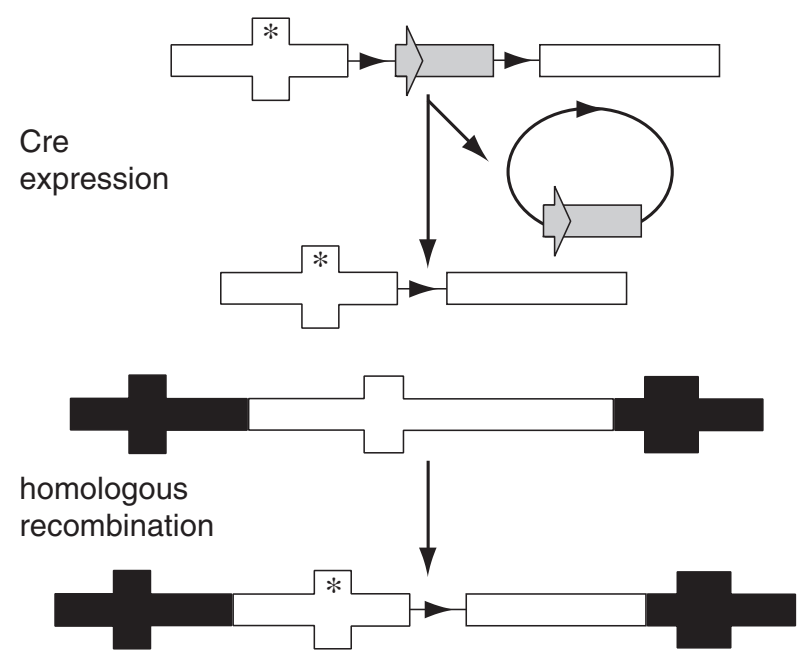

Homologous seqeunces

construct and target gene
Nonhomologous sequences

positive selectable marker

genomic sequences

lox sequence

vector sequences

promoter sequence

mutation

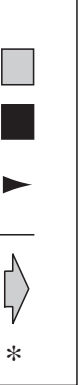

Figure 4.29.6 Using the Cre/loxP system to introduce subtle mutations. The subtle mutation is introduced along with the selectable marker in the targeting vector. The selectable marker is then removed by transient expression of $\mathrm{Cre}$, which leaves only the small lox $P$ site in the genome in a silent location.

This can be a particular problem in gene clusters where neighboring genes are in the same family, since the genes affected may have similar or identical functions. As a result, slight differences in targeting constructs have led to marked differences in phenotype.

If the targeting construct includes loxP sites flanking the neo gene, then neo can be removed after targeting by transient expression of the Cre recombinase (as discussed in Fig. 4.29.3). This will leave the small loxP site in the genomic DNA, but the construct can be engineered so that this is in an innocuous location, such as an intron. Although theoretically even a loxP site could cause alterations in the expression of neighboring genes, no such cases have yet been reported. The efficiency of Cre recombination from transient expression reported in the literature varies widely, from $\sim 2 \%$ to $\sim 15 \%$ (Sauer and Henderson, 1989; Abuin and Bradley, 1996). This rate should be distinguished from the efficiency of Cre recombina- tion in vivo, where the expression of Cre is derived from sequences integrated into the genome and therefore will show longer-lasting expression in nearly all cases.

\section{Introduction of Subtle Mutations Using Cre/loxP}

The strategy described in the previous section involves introducing subtle mutations by first duplicating sequences and then screening for intrachromosomal recombination that removes the redundant sequences and leaves the mutation. A limitation to this approach is that the second homologous recombination event occurs only infrequently. A more efficient method is to use a replacement construct containing the subtle mutation and then remove the positive selectable marker, which is flanked by loxP sites, using the Cre recombinase system (Fig. 4.29.6). This is identical in effect to removing the neo locus after gene inactivation, except that instead of an inactive gene, the
Gene Cloning, Expression, and Mutagenesis

\subsection{7}

Supplement 21 


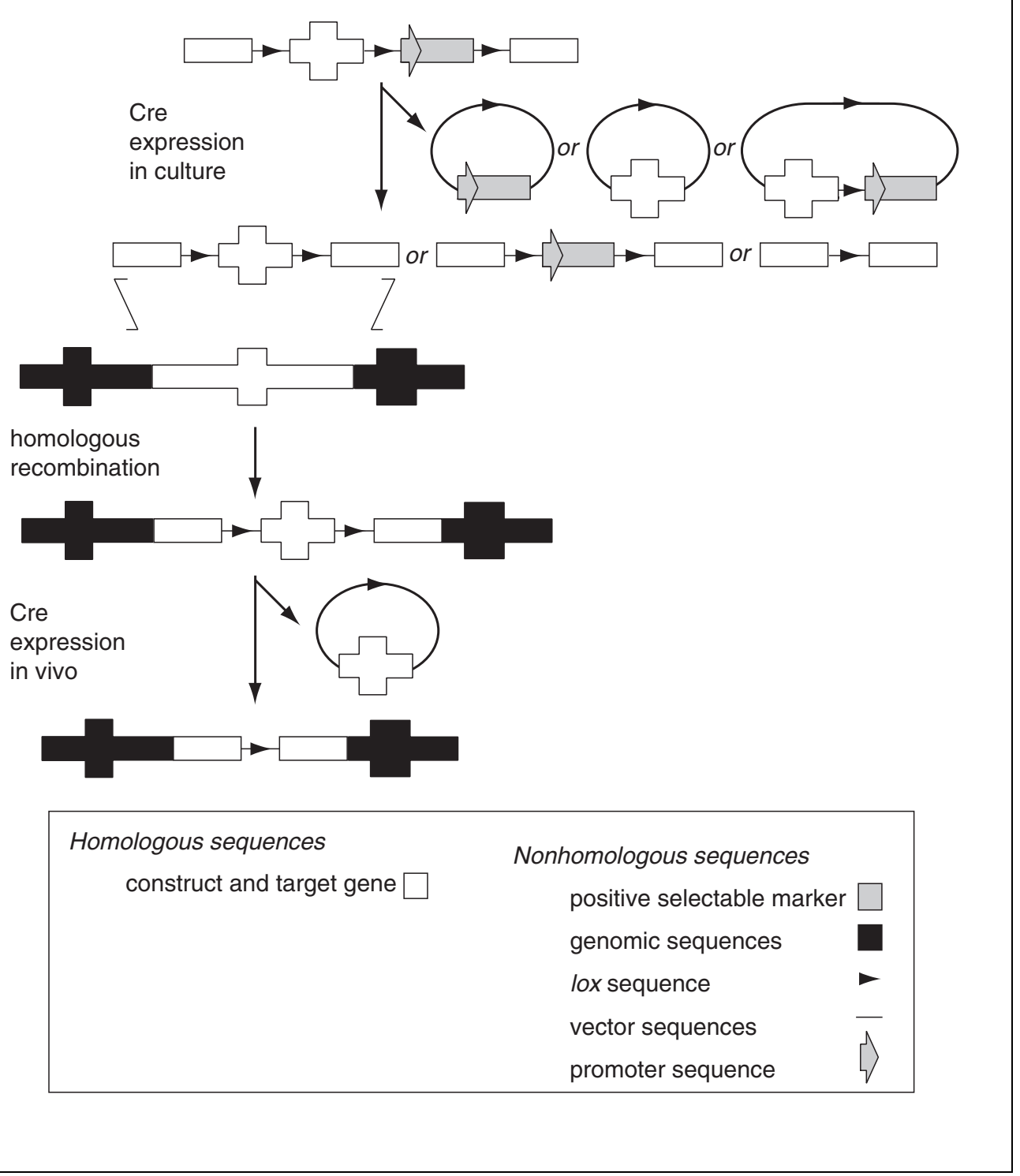

Figure 4.29.7 Conditional gene targeting using the Cre/loxP system. The targeting vector contains three loxP sites that flank the regions of the gene to be removed and the positive selectable marker neo. After homologous recombination is obtained, the selectable marker is excised from the gene by transient expression of Cre. The correct recombination is identified by screening by Southern analysis or PCR. The mutant ES cells are then used to produce a transgenic animal, which is finally bred to an animal line expressing Cre under either temporal or spatial control. Cre can also be ubiquitously expressed to obtain a knockout in all tissues, including the germ line.

replaced sequences contain a subtly mutated version.

\section{Spatial Control of Knockout}

Spatially controlled targeted gene inactivations can be performed in two ways. The most common makes use of cell type-specific promoters (sometimes called tissue-specific promoters, even though tissues are actually made of a number of different cell types). This approach begins with the creation of a transgenic animal that expresses Cre in only some cells using a cell type-restricted promoter. A second transgenic animal line is then created by homologous recombination that contains lox $P$ sites flanking a portion of the gene that is critical for activity, typically important exons (Fig. 4.29.7). Initially there are three lox $P$ sites flanking this important gene region and the selectable marker. After homologous recombination has been verified, Cre is transiently expressed, and loops out regions of DNA between pairs of loxP sites. The resultant colonies are screened for the desired recombination (loss of 
the selectable marker but retention of all regions of the gene). Depending on the frequency of recombination at the site, it may be useful to use a construct that contains a negative selectable marker (such as cytosine deaminase in the example shown in Fig. 4.29.2) between the loxP sites along with the positive selectable marker. In this way cells that have lost the markers can be selected.

The targeted line will have normal expression of the targeted gene, since its only modification is the presence of loxP sites in innocuous sites (e.g., introns). When the two lines are bred together, the Cre recombinase will loop out the DNA - inactivating the gene-only in those cells where it is expressed. In this way, tissue-specific knockouts of a number of genes have been generated (Gu et al., 1994; Agah et al., 1997). The method also has the advantage that, once a transgenic line is generated with the desired restricted expression of Cre, the approach can be applied to a number of targeted lines. In addition, it is not necessary to make separate constructs for a restricted and a complete knockout, since Cre-expressing lines have been made that will produce rearrangement in all tissues when bred to the targeted line (Schwenk et al., 1995).

Another way of spatially controlling knockout is to use an expression system for Cre that can be applied to absolute location. In some cases, no restricted expression pattern is known for a gene that matches the desired spatial alteration; in others, the site may be particularly amenable to viral manipulation (as with an epithelial or endothelial surface) or accessible by direct injection (such as sterotactic injection of the central nervous system). By using a viral vector to express the Cre protein, it is possible to obtain knockouts that are spatially limited by the viral infection. This strategy has been applied to a number of tissues including the brain, liver, colon, and heart (Rohlmann et al., 1996; Wang et al., 1996; Agah et al., 1997; Shibata et al., 1997; van der Neut, 1997).

\section{Temporal Control of Knockout}

In many cases the phenotype of interest is in the adult animal but, because the gene is necessary for development, no adult animals are obtained. Delaying the expression of Cre activity until the animal is an adult would allow normal development, and then the knockout could be created in the adult (Rajewsky et al., 1996). This can be accomplished by using a conditional expression system (e.g., the tet-on, tet-off, or ecdysone systems; see St-Onge et al.,
1996) or other inducible system (such as an interferon-inducible promoter; Kuhn et al., 1995) to express Cre at the proper time. This would, however, require the construction of animals containing three transgenes. Another approach that has been used is the creation of a fusion protein with either a modified estrogen receptor (Feil et al., 1996, 1997; Zhang et al., 1996; Brocard et al., 1997) or a modified glucocorticoid receptor (Brocard et al., 1998). These fusion proteins are inactive for recombination until the appropriate ligand is added, allowing temporal control in an animal with only transgenes. The Flp/FRT recombinase system can be used in an analogous way. Combination of the two systems can allow the production of complex schemes for gene mutation.

\section{LITERATURE CITED}

Abuin, A. and Bradley, A. 1996. Recycling selectable markers in mouse embryonic stem cells. Mol. Cell. Biol. 16:1851-1856.

Agah, R., Frenkel, P.A., French, B.A., Michael, L.H., Overbeek, P.A., and Schneider, M.D. 1997. Gene recombination in postmitotic cells. Targeted expression of Cre recombinase provokes cardiac-restricted, site-specific rearrangement in adult ventricular muscle in vivo. J. Clin. Invest. 100:169179.

Brocard, J., Warot, X., Wendling, O., Messaddeq, N., Vonesch, J.L., Chambon, P., and Metzger, D. 1997. Spatio-temporally controlled site-specific somatic mutagenesis in the mouse. Proc. Natl. Acad. Sci. U.S.A. 94:14559-14563.

Brocard, J., Feil, R., Chambon, P., and Metzger, D. 1998. A chimeric Cre recombinase inducible by synthetic, but not by natural ligands of the glucocorticoid receptor. Nucl. Acids Res. 26:40864090.

Cruz, A., Coburn, C.M., and Beverley, S.M. 1991. Double targeted gene replacement for creating null mutants. Proc. Natl. Acad. Sci. U.S.A. 88:7170-7174.

Feil, R., Brocard, J., Mascrez, B., LeMeur, M., Metzger, D., and Chambon, P. 1996. Ligand-activated site-specific recombination in mice. Proc. Natl. Acad. Sci. U.S.A. 93:10887-10890.

Feil, R., Wagner, J., Metzger, D., and Chambon, P. 1997. Regulation of Cre recombinase activity by mutated estrogen receptor ligand-binding domains. Biochem. Biophys. Res. Commun. 237:752-757.

Fiering, S., Kim, C.G., Epner, E.M., and Groudine, M. 1993. An "in-out" strategy using gene targeting and FLP recombinase for the functional dissection of complex DNA regulatory elements: Analysis of the $\beta$-globin locus control region. Proc. Natl. Acad. Sci. U.S.A. 90:8469-8473.

Fiering, S., Epner, E., Robinson, K., Zhuang, Y., Telling, A., Hu, M., Martin, D.I., Enver, T., Ley, T.J., and Groudine, M. 1995. Targeted deletion
Gene Cloning, Expression, and Mutagenesis

\subsection{9}

Supplement 21 
of $5^{\prime} \mathrm{HS} 2$ of the murine $\beta$-globin LCR reveals that it is not essential for proper regulation of the $\beta$-globin locus. Genes Dev. 9:2203-2213.

Fiering S., Bender, M.A., and Groudine, M. 1999. Analysis of mammalian cis-regulatory DNA elements by homolgous recombination. Methods Enzymol. 306:42-66.

Gu, H., Marth, J.D., Orban, P.C., Mossmann, H., and Rajewsky, K. 1994. Deletion of a DNA polymerase $\beta$ gene segment in T cells using cell typespecific gene targeting. Science 265:103-106.

Kuhn, R., Schwenk, F., Aguet, M., and Rajewsky, K. 1995. Inducible gene targeting in mice. Science 269:1427-1429.

Mansour, S.L. 1990. Gene targeting in murine embryonic stem cells: Introduction of specific alterations into the mammalian genome. Genet. Anal. Tech. Appl. 7:219-227.

Mansour, S.L., Thomas, K.R., and Capecchi, M.R. 1988. Disruption of the proto-oncogene int- 2 in mouse embryo-derived stem cells: A general strategy for targeting mutations to nonselectable genes. Nature 336:348-352.

Mansour, S.L., Thomas, K.R., Deng, C.X., and Capecchi, M.R. 1990. Introduction of a lacZ reporter gene into the mouse int- 2 locus by homologous recombination. Proc. Natl. Acad. Sci. U.S.A. 87:7688-7692.

Mombaerts, P., Clarke, A.R., Hooper, M.L., and Tonegawa, S. 1991. Creation of a large genomic deletion at the T-cell antigen receptor betasubunit locus in mouse embryonic stem cells by gene targeting. Proc. Natl. Acad. Sci. U.S.A. 88:3084-3087.

Mortensen, R.M., Zubiaur, M., Neer, E.J., and Seidman, J.G. 1991. Embryonic stem cells lacking a functional inhibitory G-protein subunit (alpha i2) produced by gene targeting of both alleles. Proc. Natl. Acad. Sci. U.S.A. 88:7036-7040.

Mortensen, R.M., Conner, D.A., Chao, S., Geisterfer, L.A., and Seidman, J.G. 1992. Production of homozygous mutant ES cells with a single targeting construct. Mol. Cell. Biol. 12:2391-2395.

Olson, E.N., Arnold, H.H., Rigby, P.W., and Wold, B.J. 1996. Know your neighbors: Three phenotypes in null mutants of the myogenic bHLH gene MRF4. Cell 85:1-4.

Pham, C.T., MacIvor, D.M., Hug, B.A., Heusel, J.W., and Ley, T.J. 1996. Long-range disruption of gene expression by a selectable marker cassette. Proc. Natl. Acad. Sci. U.S.A. 93:13090-13095.

Rajewsky, K., Gu, H., Kuhn, R., Betz, U.A., Muller, W., Roes, J., and Schwenk, F. 1996. Conditional gene targeting. J. Clin. Invest. 98:600-603.

Robertson, E.J. 1991. Using embryonic stem cells to introduce mutations into the mouse germ line. Biol. Reprod. 44:238-245.

Rohlmann, A., Gotthardt, M., Willnow, T.E., Hammer, R.E., and Herz, J. 1996. Sustained somatic gene inactivation by viral transfer of Cre recombinase. Nat. Biotechnol. 14:1562-1565.

Sauer, B. 1993. Manipulation of transgenes by sitespecific recombination: Use of Cre recombinase. Methods Enzymol. 225:890-900.

Sauer, B. and Henderson, N. 1989. Cre-stimulated recombination at loxP-containing DNA sequences placed into the mammalian genome. Nucl. Acids Res. 17:147-161.

Schwenk, F., Baron, U., and Rajewsky, K. 1995. A cre-transgenic mouse strain for the ubiquitous deletion of loxP-flanked gene segments including deletion in germ cells. Nucl. Acids Res. 23:50805081.

Shibata, H., Toyama, K., Shioya, H., Ito, M., Hirota, M., Hasegawa, S., Matsumoto, H., Takano, H., Akiyama, T., Toyoshima, K., Kanamaru, R., Kanegae, Y., Saito, I., Nakamura, Y., Shiba, K., and Noda, T. 1997. Rapid colorectal adenoma formation initiated by conditional targeting of the Apc gene. Science 278:120-123.

St-Onge, L., Furth, P.A., and Gruss, P. 1996. Temporal control of the Cre recombinase in transgenic mice by a tetracycline responsive promoter. $\mathrm{Nucl}$. Acids Res. 24:3875-3877.

te Riele, H., Maandag, E.R., Clarke, A., Hooper, M., and Berns, A. 1990. Consecutive inactivation of both alleles of the pim-1 proto-oncogene by homologous recombination in embryonic stem cells. Nature 348:649-651.

van der Neut, R. 1997. Targeted gene disruption: Applications in neurobiology. J. Neurosci. Methods 71:19-27.

Vooijs, M., van der Valk, M., te Riele, H., and Berns, A. 1998. Flp-mediated tissue-specific inactivation of the retinoblastoma tumor suppressor gene in the mouse. Oncogene 17:1-12.

Wang, Y., Krushel, L.A., and Edelman, G.M. 1996. Targeted DNA recombination in vivo using an adenovirus carrying the cre recombinase gene. Proc. Natl. Acad. Sci. U.S.A. 93:3932-3936.

Yagi, T., Ikawa, Y., Yoshida, K., Shigetani, Y., Takeda, N., Mabuchi, I., Yamamoto, T., and Aizawa, S. 1990. Homologous recombination at c-fyn locus of mouse embryonic stem cells with use of diphtheria toxin A-fragment gene in negative selection. Proc. Natl. Acad. Sci. U.S.A. 87:9918-9922.

Zhang, Y., Riesterer, C., Ayrall, A.M., Sablitzky, F., Littlewood, T.D., and Reth, M. 1996. Inducible site-directed recombination in mouse embryonic stem cells. Nucl. Acids Res. 24:543-548.

Zimmer, A. 1992. Manipulating the genome by homologous recombination in embryonic stem cells. Annu. Rev. Neurosci. 15:115-137.

\section{Contributed by Richard Mortensen \\ University of Michigan \\ Medical School \\ Ann Arbor, Michigan}

\section{Overview of Gene Targeting by Homologous Recombination}

